\title{
How Do You Work? Understanding User Needs for Responsive Study Space Design
}

\author{
Asha L. Hegde, Tricia M. Boucher, and Allison D. \\ Lavelle
}

To design learning commons study spaces responsive to user needs, the study spaces programming team at Texas State University created a survey focused on (1) how faculty and students like to work and (2) inspirational environmental attributes. Results from 199 respondents indicate that (1) 85 percent prefer to study/work alone, and (2) environmental parameters noise/sound, light, and links to nature most affect work/study space quality. Removing the concept of library from the survey provided generalized results about study/work environments, suggesting that well designed study spaces can occur anywhere on campuses that serve large populations with a single library.

\section{Introduction and Purpose}

The academic library is changing. No longer simply a warehouse of information, the university library is a place for students and patrons to study, collaborate, socialize, and learn through social activities. ${ }^{1}$ This change in use reflects a paradigmatic shift from understanding the library as information repository to understanding the library as "place," "third space," and/or "learning commons." As the paradigm of the academic library changes, it transforms academic library design. ${ }^{2}$ Because the conceptual basis of current academic library design is still changing away from traditional academic library spaces, it is necessary to continuously evaluate user needs and desires for study spaces, and what makes an ideal study space, to keep the design of academic libraries relevant to its patrons.

When it was built in 1990, the Alkek Library at Texas State University in San Marcos served a student population of 20,000. Today, Texas State University serves more than 36,000 students enrolled at the main campus. With door counts often surpassing 12,000 students per day during finals, the 3,000 seats in the seven-story library are inadequate to serve the needs of students. In response, library and university administration embarked on renovations to transform the first four floors of the library into a learning commons, providing more space-and more types of spaces - for students to gather

Asha L. Hegde is Associate Professor of Interior Design and Lighting, Tricia M. Boucher is User Experience Librarian / Psychology Librarian, and Allison D. Lavelle is a graduate student, all at Texas State University; email:ah16@txstate.edu, squirrel@txstate.edu, adl92@txstate.edu. (2018 Asha L. Hegde, Tricia M. Boucher, and Allison D. Lavelle, Kyle Siler, Juan Pablo Alperin, and Vincent Larivière, AttributionNonCommercial (http://creativecommons.org/licenses/by-nc/4.0/) CC BY-NC. 
and learn. Administration charged the Learning Commons Programming subteam focusing on study spaces with this question: What are the study, learning, and research space needs of Texas State University's faculty and students?

Texas State University is a diverse community. It is a Hispanic Serving Institution (HSI) with a growing Hispanic population (nearly 10 percent overall growth in Hispanic student enrollment since 2011). More than 50 percent of undergraduates are first-generation college students. Additionally, 81 percent of students live off-campus and need a place to spend their time between classes and activities. ${ }^{3}$ This constituency is not unique in higher education, and the team decided a general survey to a broad cross-section of students and faculty would be useful to capture all segments of the university population.

The survey asked these core questions: "how do students and faculty study/work," "what environmental attributes enhance their studying and academic work experiences," and "what creates an ideal study and work space." To remove any preconceptions or constraints in envisioning how they liked to study or work or what makes an inspiring study/work space, the team decided to remove the concept of "library" from the survey. This paper reports on the general survey, one facet of the team's total approach to exploring these questions, which informed their report to the architectural programming consultant.

This study sought to understand current user needs and desires to provide holistic evidence of study/work modes and space needs in general. While the findings of this study give direction to the library architects, the team decided to generalize these questions to respond to the university's expanding space needs. Finite library space and rapid population growth suggest a need for study/work spaces not only just in the library but also distributed across campus. Analyzed against the growth and planned program changes at Texas State University, this information supports a predictive program that will fit the needs of the university's students and faculty, both for the library and the campus.

\section{Literature Review}

During the last 30 years, the academic library paradigm shifted from "library" to "information commons" 4 to "learning commons." 5 The learning commons concept focuses less on the information found in the library and more on all the ways learning can take place within the library, with an emphasis on social and interactive modes of learning, including group studying, collaborative projects, and the creative use of maker spaces. ${ }^{6}$

While the emphasis of many built learning commons' designs are technology and social learning, which reflect recent changes in teaching and learning styles, ${ }^{7}$ the most current learning commons concepts suggest a wider variety of spaces are necessary. Beginning in 2015, the literature begins to present a more nuanced view of learning commons' spaces and their functions based on varying student needs, ${ }^{8}$ including active/ communal spaces, interactive/collaborative spaces, reading rooms, and study rooms/ alcoves. ${ }^{9}$ The most current literature about study space in the library proposes that quiet spaces in the library are needed to accommodate: diversity in learning styles, ${ }^{10}$ variety in work assigned based on major of study, ${ }^{11}$ and the studying lifecycle - changes due to year of study (from freshman to graduate student) as well as fluctuations in study styles and intensity over the course of a single academic semester. ${ }^{12}$ This evolution in the literature is based on people's general work modes depending on personal preferences and the type of work to be done; work alone, work with a group, or work alone but in group. ${ }^{13}$ Literature about office space design reflects these same concepts about work and work spaces. ${ }^{14}$

Environmental factors play a large role in how people perceive and use space, and how that space affects well-being and productivity. Additional studies address envi- 
ronmental factors such as noise, crowdedness, territory and furniture arrangements, lighting, daylight, and so forth in the study and work environments, both in libraries and in other work/study spaces. ${ }^{15}$ The need for control of noise, need for quietness, and acoustical privacy appeared as vital factors needed for successful work/study spaces. ${ }^{16}$

Visual privacy, proximity, and personal space/territory are all important to users of the library space and other work/study spaces. ${ }^{17}$ Since personalization of space is impossible for students in a library/learning commons, privacy or personal space becomes even more important, requiring a different type of control of space. ${ }^{18}$ Studies indicate that students alter furniture arrangement or orient themselves away from others to seek visual privacy and to respect personal space and territory. ${ }^{19}$ Students generally prefer not to use occupied tables even if there are seats available. ${ }^{20}$

Research indicates that attributes of electric light, specifically color temperature, support significant improvement on student cognitive performance in classroom, ${ }^{21}$ and with the use of a dynamic lighting system in a classroom, students showed improvement in concentration levels and reading. ${ }^{22}$ Daylight without glare in schools and classrooms shows positive effects on learning and test-taking performance, ${ }^{23}$ and in offices shows positive effects on human performance. ${ }^{24}$ Kilic and Hasirci studied the effects of daylighting on visual comfort of library users in relation to four environmental aspects: privacy, personal space, territoriality, and crowding. ${ }^{25}$ Their research found that daylight and these four environmental aspects were related and should be considered in design of libraries for better usage and fulfillment of user experience.

Literature regarding library/learning commons design shows the shift from emphasis only on communal/social learning spaces to an equal emphasis on quiet learning spaces. Literature about libraries and other study/work spaces also highlights the importance of several aspects of the environment that play a key role in creating a comfortable and productive space to study/work. The goal of this study survey was multifaceted and exploratory and intended to provide information to architects and design professionals to use when creating study spaces. To do this, the survey was designed to understand Texas State University's faculty and students' work modes, what kinds of work they do when in those modes, and what they need in a study space to effectively work, study, and research. It also was created to better understand what attributes Texas State University's constituents desire in an ideal study space that would help provide inspiration. Finally, the survey was conducted to find out if these modes, needs, and desires were predicted by the literature or were unique to Texas State University.

\section{Research Questions}

1. What are the work/study mode preferences - to work alone, work or study collaboratively in a group, work alone but with a group of friends/peers/colleagues - of university students and faculty?

2. What types of academic work do students and faculty engage in when involved in different work modes - work alone, work or study collaboratively in a group, work alone but with a group of friends/peers/colleagues?

3. Are there discernible differences between students and faculty in how they rate attributes of a study/work space that could improve their ability to work or study in the three different work modes?

4. What environmental attributes and words do students and faculty use to describe "great" and "terrible" study/work spaces?

5. What attributes do students and faculty consider "inspiring" in a space for them to work or study? 


\section{Method}

Sample and Research Participation: The study population consisted of the current undergraduate, graduate students, and faculty of Texas State University. The study employed a random sampling method in which less than 10 percent of the current undergraduate (app. 3,000 out of 32,000 students) and graduate students (app. 300 out of 4,000 students), about 150 full-time/part-time faculty (less than 10 percent) were randomly selected through an email distribution list provided by the university.

Using the randomized list, the team sent out a "research participation invitation" email with a cover letter indicating that it was conducting an IRB-approved research study, to understand what they-students and faculty-consider ideal innovative study spaces and technologies that inspire and enable them to study, learn, and research individually or in a group setting. The cover letter included a link to participate in the survey. The cover letter also clearly stated that participation is implied by completing the survey, and if they did not wish to participate not to answer the survey. The team received 199 survey responses, which comprises the study sample.

Instrument: Instead of looking at types of study spaces, the team first decided to look at the ways people work - alone, with a group, alone in a group. ${ }^{26}$ The team developed the survey instrument based on review of various existing library and study space surveys used by other universities and office settings. The team modeled content of some questions from the MIT library surve ${ }^{27}$ then conducted a pilot using the initial survey with 25 participants to estimate time taken to conduct the survey as well as adjust question items and terms. The final survey consisted of nineteen questions with sub-items within each question inquiring about work modes; things that will improve their ability to study/work/research within the context of working alone, working in a group, or working alone in a group. Also included were three open-ended questions. The first two asked participants to describe their best and worst study spaces and describe what made them so. The last asked participants to list attributes of spaces that inspire them to study or work. The survey was conducted using SurveyMonkey and researchers coded the received responses using numbers to differentiate respondents.

Statistical Analysis: This study uses cross tabulations to analyze and compare the results of research questions 1 (study/work mode preferences) and 2 (types of academic work done in different work modes). The team used the Kruskal-Wallis nonparametric test for independent samples test differences between students and faculty in rating of attributes that could improve their ability to work or study in the three different work modes. The team used content analysis to evaluate the open-ended questions.

\section{Results}

A total of 107 undergraduate students (4\% response rate), 35 graduate students (12\% response rate) and 56 faculty (37\% response rate) responded to the survey, for a total of 199 responses. The respondents represented a true random sample, representing all colleges and schools within the university.

Study/work mode: Question 1 asked participants what kind of work/study mode they prefer - to study alone, to study in a group/collaboratively, or to study alone but in a group. Faculty and students rated their preference for each of the work modes by selecting one of the choices (always, often, sometimes, occasionally, or never). Regarding working/studying alone mode, 164 (85\%) responded that they like to study or work alone often or always, while 14 percent of the sample (26 individuals) selected sometimes or occasionally.

For working or studying with a group (collaboratively), 21 percent of the participants (40 individuals) studied this way often or always, 63 percent (117 individuals) worked collaboratively sometimes or occasionally, while 16 percent never work or study in a 
group. With regard to the work mode "work alone but with a group of friends/peers/ colleagues," 35 (19\%) indicated that they like to study/work this way often or always, while 37 percent indicated that they never work or study this way. The faculty had the largest percentage $(68 \%, 30$ individuals) that indicated that they never work alone in a group or social setting (see table 1 ).

\begin{tabular}{|c|c|c|c|c|c|}
\hline & Stu & $\begin{array}{l}\text { TABLE } \\
\text { ly/Work Mod }\end{array}$ & 1 & & \\
\hline & & $v$ do you like to & ork or study & & \\
\hline Work Mode & & Undergraduate & Graduate & Faculty & Overall \\
\hline & & $\mathrm{n}=106$ & $\mathrm{n}=34$ & $\mathrm{n}=52$ & $\mathrm{n}=192$ \\
\hline Study/Work & Always & $29 \mid 27 \%$ & $11 \mid 32 \%$ & $26 \mid 50 \%$ & $66 \mid 34 \%$ \\
\hline Alone & Often & $60 \mid 57 \%$ & $20 \mid 59 \%$ & $18 \mid 34 \%$ & $98 \mid 51 \%$ \\
\hline & Sometimes & $12 \mid 11 \%$ & $3 \mid 9 \%$ & $2 \mid 4 \%$ & $17 \mid 9 \%$ \\
\hline & Occasionally & $5 \mid 5 \%$ & $0 \mid 0 \%$ & $4 \mid 8 \%$ & $9 \mid 5 \%$ \\
\hline & Never & $0 \mid 0 \%$ & $0 \mid 0 \%$ & $2 \mid 4 \%$ & $2 \mid 1 \%$ \\
\hline & & $\mathrm{n}=105$ & $\mathrm{n}=33$ & $\mathrm{n}=48$ & $\mathrm{n}=186$ \\
\hline Study/Work & Always & $3 \mid 3 \%$ & $0 \mid 0 \%$ & $3 \mid 6 \%$ & $6 \mid 3 \%$ \\
\hline & Often & $21 \mid 20 \%$ & $5 \mid 15 \%$ & $8 \mid 17 \%$ & $34 \mid 18 \%$ \\
\hline & Sometimes & $38 \mid 36 \%$ & $14 \mid 42.5 \%$ & $12 \mid 25 \%$ & $64 \mid 34.5 \%$ \\
\hline & Occasionally & $30 \mid 29 \%$ & $10 \mid 30.5 \%$ & $13 \mid 27 \%$ & $53 \mid 28.5 \%$ \\
\hline & Never & $13 \mid 12 \%$ & \begin{tabular}{l|l|}
$42 \%$ \\
\end{tabular} & $12 \mid 25 \%$ & $29 \mid 16 \%$ \\
\hline & & $\mathrm{n}=106$ & $\mathrm{n}=33$ & $\mathrm{n}=44$ & $\mathrm{n}=183$ \\
\hline Study/Work & Always & $3 \mid 3 \%$ & $1 \mid 3 \%$ & $1 \mid 2 \%$ & $5 \mid 3 \%$ \\
\hline Alone but in a & Often & $19 \mid 18 \%$ & $7 \mid 21 \%$ & $4 \mid 9 \%$ & $30 \mid 16 \%$ \\
\hline & Sometimes & $25 \mid 24 \%$ & $10 \mid 30.5 \%$ & $3 \mid 7 \%$ & $38 \mid 21.5 \%$ \\
\hline & Occasionally & $31 \mid 29 \%$ & $4 \mid 12 \%$ & $6 \mid 14 \%$ & $41 \mid 22 \%$ \\
\hline & Never & $28 \mid 26 \%$ & $11 \mid 33.5 \%$ & $30 \mid 68 \%$ & $69 \mid 37.5 \%$ \\
\hline
\end{tabular}

Types of work: Question 2 asked participants what kinds of academic work are conducted by students and faculty when involved in different work modes - work alone, work in a group/collaboratively, or work alone but in a group setting. Survey results showed that when "working alone," a large percentage of the undergraduate students worked on homework (91\%), studying (92\%), and writing (78\%). Results also indicate that a large percentage of the graduate students write $(97 \%)$, do research-related work $(86 \%)$, study $(83 \%)$, do class-related work $(74 \%)$, and participate in project preparation and presentation when working alone. Research (89\%) and writing (76\%) are the top two things that faculty do when working alone (see table 2).

More than 85 percent of the graduate and undergraduate students reported that they work collaboratively (with a group) when working on project preparation and presentation. Undergraduates like to study (62\%) and do homework (57\%) with a group of friends even when doing individual work (see table 2).

Work space attributes: Research question 3 asked participants to rate attributes of a study/work space that could improve their ability to work or study in the three different 


\begin{tabular}{|c|c|c|c|c|c|}
\hline \multicolumn{6}{|c|}{$\begin{array}{c}\text { TABLE } 2 \\
\text { Academic Work Done When Involved in Different Study/Work Modes }\end{array}$} \\
\hline \multicolumn{6}{|c|}{ (\# of respondents, \%) } \\
\hline Work Modes & Kinds of Work & Undergraduate & Graduate & Faculty & Overall \\
\hline \multirow{6}{*}{$\begin{array}{l}\text { Study/Work } \\
\text { Alone }\end{array}$} & Write & $83 \mid 78 \%$ & $34 \mid 97 \%$ & $41 \mid 76 \%$ & $158 \mid 81 \%$ \\
\hline & Research & $70 \mid 66 \%$ & $30 \mid 86 \%$ & $48 \mid 89 \%$ & $148 \mid 76 \%$ \\
\hline & Study & $98 \mid 92 \%$ & $29 \mid 83 \%$ & $4 \mid 7 \%$ & $131 \mid 67 \%$ \\
\hline & $\begin{array}{l}\text { Homework/ } \\
\text { classwork }\end{array}$ & $96 \mid 91 \%$ & $26 \mid 74 \%$ & $2 \mid 4 \%$ & $124 \mid 64 \%$ \\
\hline & $\begin{array}{l}\text { Prepare projects/ } \\
\text { presentations }\end{array}$ & $52 \mid 49 \%$ & $25 \mid 71 \%$ & $25 \mid 46 \%$ & $102 \mid 52 \%$ \\
\hline & N/A & $0 \mid 0 \%$ & $0 \mid 0 \%$ & $2 \mid 4 \%$ & $2 \mid 1 \%$ \\
\hline \multirow{6}{*}{$\begin{array}{l}\text { Study/Work } \\
\text { in a Group }\end{array}$} & Write & $13 \mid 12 \%$ & $5 \mid 14 \%$ & $16 \mid 30 \%$ & $34 \mid 17 \%$ \\
\hline & Research & $37 \mid 35 \%$ & $14 \mid 40 \%$ & $23 \mid 43 \%$ & $74 \mid 38 \%$ \\
\hline & Study & $70 \mid 65 \%$ & $16 \mid 46 \%$ & $4 \mid 7 \%$ & $90 \mid 46 \%$ \\
\hline & $\begin{array}{l}\text { Homework/ } \\
\text { classwork }\end{array}$ & $35 \mid 33 \%$ & $7 \mid 20 \%$ & $0 \mid 0 \%$ & $42 \mid 21 \%$ \\
\hline & $\begin{array}{l}\text { Prepare projects/ } \\
\text { presentations }\end{array}$ & $94 \mid 88 \%$ & $31 \mid 89 \%$ & $25 \mid 46 \%$ & $150 \mid 77 \%$ \\
\hline & N/A & $4 \mid 4 \%$ & $2 \mid 6 \%$ & $14 \mid 26 \%$ & $20 \mid 10 \%$ \\
\hline \multirow{6}{*}{$\begin{array}{l}\text { Study/Work } \\
\text { Alone but } \\
\text { with a Group }\end{array}$} & Write & $33 \mid 32 \%$ & $11 \mid 31 \%$ & $11 \mid 20 \%$ & $55 \mid 29 \%$ \\
\hline & Research & $32 \mid 31 \%$ & $16 \mid 46 \%$ & $14 \mid 26 \%$ & $62 \mid 32 \%$ \\
\hline & Study & $65 \mid 63 \%$ & $16 \mid 46 \%$ & $2 \mid 4 \%$ & $83 \mid 43 \%$ \\
\hline & $\begin{array}{l}\text { Homework/ } \\
\text { classwork }\end{array}$ & $60 \mid 58 \%$ & $16 \mid 46 \%$ & $2 \mid 4 \%$ & $78 \mid 40 \%$ \\
\hline & $\begin{array}{l}\text { Prepare projects/ } \\
\text { presentations }\end{array}$ & $31 \mid 30 \%$ & $15 \mid 43 \%$ & $13 \mid 24 \%$ & $59 \mid 31 \%$ \\
\hline & N/A & $21 \mid 20 \%$ & $9 \mid 26 \%$ & $30 \mid 56 \%$ & $60 \mid 31 \%$ \\
\hline
\end{tabular}

work modes. The team also compared the three groups (undergraduates, graduates, and faculty) to discern differences between students and faculty in how they rate the attributes. For each work mode (work alone, work or study collaboratively in a group, work alone but with a group of friends/peers or colleagues), participants rated $15 \mathrm{items}$ that could improve their ability to work or study on a scale of $1=$ very undesirable, 2 $=$ undesirable, $3=$ neutral, $4=$ desirable, and $5=$ very desirable.

The overall median score for attributes under study/work alone fell between 3.0 and 5.0 (see table 3). The overall top items for this work mode receiving a median of 5.0 were: varied seating options, charging stations, natural light, and quiet.

Even though "conference capabilities" median rating was 3.0 (neutral score) for undergraduates and graduates, faculty rated it higher (4.0). A Kruskal-Wallis test shows that there are significant differences for "conference capabilities" among groups, $\chi^{2}(2)$ $=21.26, P=0.00$. A Kruskal-Wallis test shows that there are significant differences for "food/café" between groups, $\chi^{2}(2)=6.923, P=0.031$ (see table 4 ). The median score for "increased safety locker/spaces with ID entry" for graduate students is 5.0, while the undergraduates and faculty rated at 4.0. A Kruskal-Wallis test shows that there 
How Do You Work? 901

\begin{tabular}{|c|c|c|c|c|}
\hline \multicolumn{5}{|c|}{$\begin{array}{c}\text { TABLE } 3 \\
\text { Median Responses from Undergraduates, Graduates and Faculty for Study/ } \\
\text { Work Alone }\end{array}$} \\
\hline \multicolumn{5}{|c|}{ Study/Work Alone } \\
\hline & \multicolumn{4}{|c|}{ Median } \\
\hline Attributes & Undergraduate & Graduate & Faculty & Overall \\
\hline Varied Seating Options & 5.0 & 5.0 & 4.0 & 5.0 \\
\hline Printing Options & 4.0 & 4.0 & 4.0 & 4.0 \\
\hline Charging Stations & 5.0 & 5.0 & 5.0 & 5.0 \\
\hline Media Players & 3.0 & 3.0 & 3.0 & 3.0 \\
\hline Writable Surfaces & 4.0 & 4.0 & 4.0 & 4.0 \\
\hline Conference Capabilities & 3.0 & 3.0 & 4.0 & 3.0 \\
\hline Food/Cafe & 4.0 & 4.0 & 4.0 & 4.0 \\
\hline Increased Safety & 4.0 & 5.0 & 4.0 & 4.0 \\
\hline Natural Light & 5.0 & 5.0 & 5.0 & 5.0 \\
\hline Quiet & 5.0 & 5.0 & 5.0 & 5.0 \\
\hline Background Noise & 3.0 & 3.5 & 2.0 & 3.0 \\
\hline Separate Room & 4.0 & 4.0 & 4.0 & 4.0 \\
\hline Partially Closed Off & 4.0 & 4.0 & 4.0 & 4.0 \\
\hline Against a Wall & 4.0 & 4.0 & 3.0 & 4.0 \\
\hline Out in the Open & 3.0 & 3.0 & 2.0 & 3.0 \\
\hline
\end{tabular}

\begin{tabular}{|l|l|c|c|l|c|}
\hline \multicolumn{7}{|c|}{ TABLE 4 } \\
Nonparametric Kruskal-Wallis Test for Study/Work Alone \\
\hline \multicolumn{7}{|c|}{ Study/Work Alone Ranks } \\
\hline Attributes & Groups & N & Mean Rank & \multicolumn{2}{c|}{ Test Statistics } \\
\hline Varied Seating Options & Undergraduate & 107 & 102.94 & Chi-Square & 4.6 \\
\hline & Graduate & 35 & 102.34 & df & 2 \\
\hline & Faculty & 53 & 85.16 & Asymp. Sig. & 0.1 \\
\hline & Total & 195 & & & \\
\hline Printing Options* & Undergraduate & 107 & 102.5 & Chi-Square & 9.464 \\
\hline & Graduate & 35 & 112.01 & df & 2 \\
\hline & Faculty & 53 & 79.67 & Asymp. Sig. & 0.009 \\
\hline & Total & 195 & & & \\
\hline Charging Stations & Undergraduate & 107 & 98.69 & Chi-Square & 0.558 \\
\hline & Graduate & 34 & 99.84 & df & 2 \\
\hline & Faculty & 53 & 93.59 & Asymp. Sig. & 0.756 \\
\hline & Total & 194 & & & \\
\hline Media Players & Undergraduate & 107 & 102.4 & Chi-Square & 1.71 \\
\hline & Graduate & 35 & 94.5 & df & 2 \\
\hline & Faculty & 53 & 91.42 & Asymp. Sig. & 0.425 \\
\hline & Total & 195 & & & \\
\hline & & & & &
\end{tabular}




\begin{tabular}{|c|c|c|c|c|c|}
\hline \multicolumn{6}{|c|}{$\begin{array}{c}\text { TABLE } 4 \\
\text { Nonparametric Kruskal-Wallis Test for Study/Work Alone }\end{array}$} \\
\hline \multicolumn{6}{|c|}{ Study/Work Alone Ranks } \\
\hline Attributes & Groups & $\mathrm{N}$ & Mean Rank & \multicolumn{2}{|c|}{ Test Statistics } \\
\hline \multirow[t]{4}{*}{ Writable Surfaces } & Undergraduate & 107 & 104.13 & Chi-Square & 4.506 \\
\hline & Graduate & 34 & 82.75 & df & 2 \\
\hline & Faculty & 53 & 93.58 & Asymp. Sig. & 0.105 \\
\hline & Total & 194 & & & \\
\hline \multirow[t]{4}{*}{ Conference Capabilities* } & Undergraduate & 107 & 85.64 & Chi-Square & 21.26 \\
\hline & Graduate & 35 & 94.84 & $\mathrm{df}$ & 2 \\
\hline & Faculty & 53 & 125.04 & Asymp. Sig. & 0 \\
\hline & Total & 195 & & & \\
\hline \multirow[t]{4}{*}{ Food/Café* } & Undergraduate & 107 & 102.71 & Chi-Square & 6.923 \\
\hline & Graduate & 35 & 107.8 & df & 2 \\
\hline & Faculty & 53 & 82.01 & Asymp. Sig. & 0.031 \\
\hline & Total & 195 & & & \\
\hline \multirow[t]{4}{*}{ Increased Safety* } & Undergraduate & 106 & 98.52 & Chi-Square & 7.057 \\
\hline & Graduate & 35 & 116.13 & $\mathrm{df}$ & 2 \\
\hline & Faculty & 54 & 85.22 & Asymp. Sig. & 0.029 \\
\hline & Total & 195 & & & \\
\hline \multirow[t]{4}{*}{ Natural Light } & Undergraduate & 107 & 95.31 & Chi-Square & 1.031 \\
\hline & Graduate & 35 & 102.23 & df & 2 \\
\hline & Faculty & 54 & 102.41 & Asymp. Sig. & 0.597 \\
\hline & Total & 196 & & & \\
\hline \multirow[t]{4}{*}{ Quiet } & Undergraduate & 107 & 93.56 & Chi-Square & 2.697 \\
\hline & Graduate & 35 & 106.19 & $\mathrm{df}$ & 2 \\
\hline & Faculty & 54 & 103.31 & Asymp. Sig. & 0.26 \\
\hline & Total & 196 & & & \\
\hline \multirow[t]{4}{*}{ Background Noise* } & Undergraduate & 107 & 96.57 & Chi-Square & 8.842 \\
\hline & Graduate & 34 & 120.34 & $\mathrm{df}$ & 2 \\
\hline & Faculty & 53 & 84.74 & Asymp. Sig. & 0.012 \\
\hline & Total & 194 & & & \\
\hline \multirow[t]{4}{*}{ Separate Room } & Undergraduate & 107 & 101.74 & Chi-Square & 3.309 \\
\hline & Graduate & 35 & 103.54 & df & 2 \\
\hline & Faculty & 53 & 86.79 & Asymp. Sig. & 0.191 \\
\hline & Total & 195 & & & \\
\hline \multirow[t]{4}{*}{ Partially Closed Off* } & Undergraduate & 107 & 98.05 & Chi-Square & 6.481 \\
\hline & Graduate & 35 & 115.93 & $\mathrm{df}$ & 2 \\
\hline & Faculty & 53 & 86.06 & Asymp. Sig. & 0.039 \\
\hline & Total & 195 & & & \\
\hline
\end{tabular}




\begin{tabular}{|l|l|c|c|l|c|}
\hline \multicolumn{7}{|c|}{ TABLE 4 } \\
Nonparametric Kruskal-Wallis Test for Study/Work Alone \\
\hline \multicolumn{7}{|c|}{ Study/Work Alone Ranks } \\
\hline Attributes & Groups & N & Mean Rank & \multicolumn{2}{c|}{ Test Statistics } \\
\hline Against a Wall* & Undergraduate & 107 & 98.14 & Chi-Square & 7.668 \\
\hline & Graduate & 35 & 117.47 & df & 2 \\
\hline & Faculty & 53 & 84.87 & Asymp. Sig. & 0.022 \\
\hline & Total & 195 & & & \\
\hline Out in the Open & Undergraduate & 107 & 98.75 & Chi-Square & 5.616 \\
\hline & Graduate & 35 & 111.13 & df & 2 \\
\hline & Faculty & 51 & 83.64 & Asymp. Sig. & 0.06 \\
\hline & Total & 193 & & & \\
\hline Notes: * Significant at $P<.05$ & & & & \\
\hline
\end{tabular}

are significant differences for "increased safety locker/spaces with ID entry" between groups, $\chi^{2}(2)=7.057, P=0.029$ (see table 4 ). "Background noise" was yet another attribute that received low desirability rating. Graduate students median score is 3.5 compared to undergraduates (3.0) and faculty (2.0). A Kruskal-Wallis test shows that there are significant differences for "background noise" between groups, $\chi^{2}(2)=8.842$, $P=0.012$ (see table 4).

\begin{tabular}{|l|l|c|c|c|}
\hline \multicolumn{5}{|c|}{ TABLE 5 } \\
Median Responses from Undergraduates, Graduates and Faculty for Study/ \\
Work in a Group \\
\hline & \multicolumn{4}{|c|}{ Study/Work in a Group } \\
\hline & \multicolumn{4}{|c|}{ Median } \\
\hline Attributes & Undergraduate & Graduate & Faculty & Overall \\
\hline Varied Seating Options & 5.0 & 5.0 & 5.0 & 5.0 \\
\hline Printing Options & 5.0 & 4.0 & 4.0 & 4.0 \\
\hline Charging Stations & 5.0 & 5.0 & 5.0 & 5.0 \\
\hline Media Players & 4.0 & 4.0 & 4.0 & 4.0 \\
\hline Writable Surfaces & 5.0 & 5.0 & 5.0 & 5.0 \\
\hline Conference Capabilities & 3.0 & 3.5 & 4.0 & 4.0 \\
\hline Food/Cafe & 4.0 & 4.0 & 4.0 & 4.0 \\
\hline Increased Safety & 4.0 & 4.0 & 3.0 & 4.0 \\
\hline Natural Light & 5.0 & 5.0 & 5.0 & 5.0 \\
\hline Quiet & 4.0 & 4.0 & 4.0 & 4.0 \\
\hline Background Noise & 3.0 & 3.0 & 2.0 & 3.0 \\
\hline Separate Room & 5.0 & 5.0 & 5.0 & 5.0 \\
\hline Partially Closed Off & 4.0 & 4.0 & 3.0 & 4.0 \\
\hline Against a Wall & 3.0 & 3.0 & 3.0 & 3.0 \\
\hline Out in the Open & 3.0 & 3.0 & 2.0 & 3.0 \\
\hline
\end{tabular}




\begin{tabular}{|c|c|c|c|c|c|}
\hline \multicolumn{6}{|c|}{$\begin{array}{c}\text { TABLE } 6 \\
\text { Nonparametric Kruskal-Wallis Test for Study/Work in a Group }\end{array}$} \\
\hline \multicolumn{6}{|c|}{ Study/Work in a Group Ranks } \\
\hline Attributes & Groups & $\mathrm{N}$ & Mean Rank & \multicolumn{2}{|c|}{ Test Statistics } \\
\hline \multirow[t]{4}{*}{ Varied Seating Options* } & Undergraduate & 104 & 104.38 & Chi-Square & 7.152 \\
\hline & Graduate & 35 & 88.59 & $\mathrm{df}$ & 2 \\
\hline & Faculty & 52 & 84.24 & Asymp. Sig. & 0.028 \\
\hline & Total & 191 & & & \\
\hline \multirow[t]{4}{*}{ Printing Options* } & Undergraduate & 104 & 106.13 & Chi-Square & 12.32 \\
\hline & Graduate & 35 & 99.41 & df & 2 \\
\hline & Faculty & 53 & 75.69 & Asymp. Sig. & 0.002 \\
\hline & Total & 192 & & & \\
\hline \multirow[t]{4}{*}{ Charging Stations } & Undergraduate & 104 & 100.32 & Chi-Square & 2.963 \\
\hline & Graduate & 35 & 96.54 & df & 2 \\
\hline & Faculty & 52 & 87 & Asymp. Sig. & 0.227 \\
\hline & Total & 191 & & & \\
\hline \multirow[t]{4}{*}{ Media Players } & Undergraduate & 103 & 102.25 & Chi-Square & 4.417 \\
\hline & Graduate & 35 & 96.41 & df & 2 \\
\hline & Faculty & 53 & 83.58 & Asymp. Sig. & 0.11 \\
\hline & Total & 191 & & & \\
\hline \multirow[t]{4}{*}{ Writable Surfaces } & Undergraduate & 104 & 100.39 & Chi-Square & 2.617 \\
\hline & Graduate & 35 & 85.41 & df & 2 \\
\hline & Faculty & 53 & 96.19 & Asymp. Sig. & 0.27 \\
\hline & Total & 192 & & & \\
\hline \multirow[t]{4}{*}{ Conference Capabilities* } & Undergraduate & 104 & 88.96 & Chi-Square & 7.468 \\
\hline & Graduate & 34 & 89.37 & df & 2 \\
\hline & Faculty & 52 & 112.6 & Asymp. Sig. & 0.024 \\
\hline & Total & 190 & & & \\
\hline \multirow[t]{4}{*}{ Food/Café* } & Undergraduate & 104 & 105.05 & Chi-Square & 9.937 \\
\hline & Graduate & 35 & 99.91 & $\mathrm{df}$ & 2 \\
\hline & Faculty & 53 & 77.46 & Asymp. Sig. & 0.007 \\
\hline & Total & 192 & & & \\
\hline \multirow[t]{4}{*}{ Increased Safety* } & Undergraduate & 104 & 105.21 & Chi-Square & 13.397 \\
\hline & Graduate & 35 & 105.03 & df & 2 \\
\hline & Faculty & 53 & 73.77 & Asymp. Sig. & 0.001 \\
\hline & Total & 192 & & & \\
\hline \multirow[t]{4}{*}{ Natural Light } & Undergraduate & 104 & 95.32 & Chi-Square & 0.545 \\
\hline & Graduate & 34 & 101.5 & $\mathrm{df}$ & 2 \\
\hline & Faculty & 53 & 93.81 & Asymp. Sig. & 0.761 \\
\hline & Total & 191 & & & \\
\hline
\end{tabular}




\begin{tabular}{|c|c|c|c|c|c|}
\hline \multicolumn{6}{|c|}{$\begin{array}{c}\text { TABLE } 6 \\
\text { Nonparametric Kruskal-Wallis Test for Study/Work in a Group }\end{array}$} \\
\hline \multicolumn{6}{|c|}{ Study/Work in a Group Ranks } \\
\hline Attributes & Groups & $\mathrm{N}$ & Mean Rank & \multicolumn{2}{|c|}{ Test Statistics } \\
\hline \multirow[t]{4}{*}{ Quiet } & Undergraduate & 103 & 97.09 & Chi-Square & 0.225 \\
\hline & Graduate & 34 & 94.75 & df & 2 \\
\hline & Faculty & 53 & 92.9 & Asymp. Sig. & 0.894 \\
\hline & Total & 190 & & & \\
\hline \multirow[t]{4}{*}{ Background Noise* } & Undergraduate & 103 & 102.59 & Chi-Square & 12.311 \\
\hline & Graduate & 35 & 109.39 & $\mathrm{df}$ & 2 \\
\hline & Faculty & 53 & 74.35 & Asymp. Sig. & 0.002 \\
\hline & Total & 191 & & & \\
\hline \multirow[t]{4}{*}{ Separate Room } & Undergraduate & 103 & 94.26 & Chi-Square & 3.504 \\
\hline & Graduate & 35 & 109.83 & $\mathrm{df}$ & 2 \\
\hline & Faculty & 53 & 90.25 & Asymp. Sig. & 0.173 \\
\hline & Total & 191 & & & \\
\hline \multirow[t]{4}{*}{ Partially Closed Off* } & Undergraduate & 103 & 101.92 & Chi-Square & 6.058 \\
\hline & Graduate & 34 & 99.68 & $\mathrm{df}$ & 2 \\
\hline & Faculty & 53 & 80.35 & Asymp. Sig. & 0.048 \\
\hline & Total & 190 & & & \\
\hline \multirow[t]{4}{*}{ Against a Wall* } & Undergraduate & 103 & 101.35 & Chi-Square & 7.487 \\
\hline & Graduate & 34 & 103.66 & df & 2 \\
\hline & Faculty & 53 & 78.89 & Asymp. Sig. & 0.024 \\
\hline & Total & 190 & & & \\
\hline \multirow[t]{4}{*}{ Out in the Open* } & Undergraduate & 103 & 104.28 & Chi-Square & 9.944 \\
\hline & Graduate & 34 & 99.1 & $\mathrm{df}$ & 2 \\
\hline & \begin{tabular}{|l|} 
Faculty \\
\end{tabular} & 53 & 76.12 & Asymp. Sig. & 0.007 \\
\hline & Total & 190 & & & \\
\hline \multicolumn{6}{|c|}{ Notes: *Significant at $P<.05$} \\
\hline
\end{tabular}

The overall median ratings for studying and working in a group-collaboratively fell between 3.0 and 5.0 (see table 5). The overall top five items receiving a median of 5.0 are: varied seating options, charging stations, writable surfaces, natural light, and separate room.

The Kruskal-Wallis test revealed significant differences among groups-undergraduates, graduates, and faculty - for nine of the fifteen attributes, namely: varied seating options; more printing options; conference capabilities; food/café; increased safety; background noise; partially closed off; against wall; and out in the open (see table 6). The overall neutral media rating received for "out in the open" (3.0) and "background noise" (3.0) by graduate students and undergraduate students differed from the median faculty rating of 2.0. Kruskal-Wallis test shows that there are significant differences for attribute "out in the open" between groups, $\chi^{2}(2)=9.944, P=0.007$ and significant differences for attribute "background noise" between groups, $\chi^{2}(2)=12.311, P=0.002$. 
Just as in the work/study alone mode, when working collaboratively, the faculty median rating for "conference capabilities" is higher (4.0) than undergraduates (3.0) and graduate students (3.5). Kruskal-Wallis test shows that there are significant differences for attribute "conference capabilities" between groups, $\chi^{2}(2)=7.468, P=0.024$ (see table 6).

Finally, in the work mode of working alone but with a group of friends/peers/colleagues, the overall median rating for all items fell between 3.0 and 5.0 (see table 7). Two attributes, varied seating options and "charging stations," received top median rating of 5.0.

Kruskal-Wallis tests indicate significant differences between groups for all attributes. A closer look at the median and mean median rates indicate that the faculty ratings fell between 1.0 and 3.5, which is much lower than the students' ratings (see table 8). Since 68 percent of the faculty indicate that they never work alone in a group setting, these low ratings and the significance differences seem to be justified.

Across all three modes, the two items that received the lowest rating (undesirable to neutral) of 3.0 are "working out in the open" and "background noise." The three attributes that received high median ratings of desirability that appear across the three study/work modes are charging stations (5.0), varied seating options (5.0), and natural light (between 4.5 and 5.0).

Attributes of worst, best and inspiring study/work spaces: The open-ended question asked participants to name the worst place they have studied or worked and then asked them to describe what made it "terrible" to study or work in. A total of $165(83 \%)$ individuals responded to this question. Respondents named specific locations and floors within the library, spaces within their department/building, home/dorm room, and coffee

\begin{tabular}{|c|c|c|c|c|}
\hline \multicolumn{5}{|c|}{$\begin{array}{c}\text { TABLE } 7 \\
\text { Median Responses from Undergraduates, Graduates and Faculty for Study/ } \\
\text { Work Alone in a Group }\end{array}$} \\
\hline \multicolumn{5}{|c|}{ Study/Work Alone in a Group } \\
\hline & \multicolumn{4}{|c|}{ Median } \\
\hline Attributes & Undergraduate & Graduate & Faculty & Overall \\
\hline Varied Seating Options & 5.0 & 4.5 & 3.5 & 5.0 \\
\hline Printing Options & 4.0 & 4.0 & 3.0 & 4.0 \\
\hline Charging Stations & 5.0 & 5.0 & 3.5 & 5.0 \\
\hline Media Players & 3.0 & 3.0 & 2.5 & 3.0 \\
\hline Writable Surfaces & 4.0 & 4.0 & 3.0 & 4.0 \\
\hline Conference Capabilities & 3.0 & 3.0 & 3.0 & 3.0 \\
\hline Food/Cafe & 4.0 & 4.0 & 2.0 & 4.0 \\
\hline Increased Safety & 4.0 & 4.0 & 3.0 & 4.0 \\
\hline Natural Light & 5.0 & 4.5 & 3.0 & 4.0 \\
\hline Quiet & 4.0 & 4.0 & 3.0 & 4.0 \\
\hline Background Noise & 3.0 & 3.0 & 1.0 & 3.0 \\
\hline Separate Room & 4.0 & 4.0 & 3.0 & 4.0 \\
\hline Partially Closed Off & 3.0 & 4.0 & 3.0 & 3.0 \\
\hline Against a Wall & 3.0 & 4.0 & 1.5 & 3.0 \\
\hline Out in the Open & 3.0 & 3.0 & 1.0 & 3.0 \\
\hline
\end{tabular}


How Do You Work? 907

\begin{tabular}{|c|c|c|c|c|c|}
\hline \multicolumn{6}{|c|}{$\begin{array}{c}\text { TABLE } 8 \\
\text { Nonparametric Kruskal-Wallis Test for Study/Work Alone in a Group }\end{array}$} \\
\hline \multicolumn{6}{|c|}{ Study/Work Alone in a Group Ranks } \\
\hline Attributes & Groups & $\mathrm{N}$ & Mean Rank & \multicolumn{2}{|c|}{ Test Statistics } \\
\hline \multirow[t]{4}{*}{ Varied Seating Options* } & Undergraduate & 107 & 102.94 & Chi-Square & 18.807 \\
\hline & Graduate & 35 & 102.34 & df & 2 \\
\hline & Faculty & 53 & 85.16 & Asymp. Sig. & 0 \\
\hline & Total & 195 & & & \\
\hline \multirow[t]{4}{*}{ Printing Options* } & Undergraduate & 107 & 102.5 & Chi-Square & 19.259 \\
\hline & Graduate & 35 & 112.01 & df & 2 \\
\hline & Faculty & 53 & 79.67 & Asymp. Sig. & 0 \\
\hline & Total & 195 & & & \\
\hline \multirow[t]{4}{*}{ Charging Stations* } & Undergraduate & 107 & 98.69 & Chi-Square & 15.874 \\
\hline & Graduate & 34 & 99.84 & df & 2 \\
\hline & Faculty & 53 & 93.59 & Asymp. Sig. & 0 \\
\hline & Total & 194 & & & \\
\hline \multirow[t]{4}{*}{ Media Players* } & Undergraduate & 107 & 102.4 & Chi-Square & 11.667 \\
\hline & Graduate & 35 & 94.5 & df & 2 \\
\hline & Faculty & 53 & 91.42 & Asymp. Sig. & 0.003 \\
\hline & Total & 195 & & & \\
\hline \multirow[t]{4}{*}{ Writable Surfaces* } & Undergraduate & 107 & 104.13 & Chi-Square & 12.627 \\
\hline & Graduate & 34 & 82.75 & df & 2 \\
\hline & Faculty & 53 & 93.58 & Asymp. Sig. & 0.002 \\
\hline & Total & 194 & & & \\
\hline \multirow[t]{4}{*}{ Conference Capabilities* } & Undergraduate & 107 & 85.64 & Chi-Square & 8.297 \\
\hline & Graduate & 35 & 94.84 & $\mathrm{df}$ & 2 \\
\hline & Faculty & 53 & 125.04 & Asymp. Sig. & 0.016 \\
\hline & Total & 195 & & & \\
\hline \multirow[t]{4}{*}{ Food/Café* } & Undergraduate & 107 & 102.71 & Chi-Square & 21.14 \\
\hline & Graduate & 35 & 107.8 & df & 2 \\
\hline & Faculty & 53 & 82.01 & Asymp. Sig. & 0 \\
\hline & Total & 195 & & & \\
\hline \multirow[t]{4}{*}{ Increased Safety* } & Undergraduate & 106 & 98.52 & Chi-Square & 18.908 \\
\hline & Graduate & 35 & 116.13 & $\mathrm{df}$ & 2 \\
\hline & Faculty & 54 & 85.22 & Asymp. Sig. & 0 \\
\hline & Total & 195 & & & \\
\hline \multirow[t]{4}{*}{ Natural Light* } & Undergraduate & 107 & 95.31 & Chi-Square & 15.697 \\
\hline & Graduate & 35 & 102.23 & $\mathrm{df}$ & 2 \\
\hline & Faculty & 54 & 102.41 & Asymp. Sig. & 0 \\
\hline & Total & 196 & & & \\
\hline
\end{tabular}




\begin{tabular}{|c|c|c|c|c|c|}
\hline \multicolumn{6}{|c|}{$\begin{array}{c}\text { TABLE } 8 \\
\text { Nonparametric Kruskal-Wallis Test for Study/Work Alone in a Group }\end{array}$} \\
\hline \multicolumn{6}{|c|}{ Study/Work Alone in a Group Ranks } \\
\hline Attributes & Groups & $\mathrm{N}$ & Mean Rank & \multicolumn{2}{|c|}{ Test Statistics } \\
\hline \multirow[t]{4}{*}{ Quiet* } & Undergraduate & 107 & 93.56 & Chi-Square & 8.403 \\
\hline & Graduate & 35 & 106.19 & df & 2 \\
\hline & Faculty & 54 & 103.31 & Asymp. Sig. & 0.015 \\
\hline & Total & 196 & & & \\
\hline \multirow[t]{4}{*}{ Background Noise* } & Undergraduate & 107 & 96.57 & Chi-Square & 30.394 \\
\hline & Graduate & 34 & 120.34 & df & 2 \\
\hline & Faculty & 53 & 84.74 & Asymp. Sig. & 0 \\
\hline & Total & 194 & & & \\
\hline \multirow[t]{4}{*}{ Separate Room* } & Undergraduate & 107 & 101.74 & Chi-Square & 19.42 \\
\hline & Graduate & 35 & 103.54 & df & 2 \\
\hline & Faculty & 53 & 86.79 & Asymp. Sig. & 0 \\
\hline & Total & 195 & & & \\
\hline \multirow[t]{4}{*}{ Partially Closed Off* } & Undergraduate & 107 & 98.05 & Chi-Square & 15.204 \\
\hline & Graduate & 35 & 115.93 & $\mathrm{df}$ & 2 \\
\hline & Faculty & 53 & 86.06 & Asymp. Sig. & 0 \\
\hline & Total & 195 & & & \\
\hline \multirow[t]{4}{*}{ Against a Wall* } & Undergraduate & 107 & 98.14 & Chi-Square & 26.279 \\
\hline & Graduate & 35 & 117.47 & df & 2 \\
\hline & Faculty & 53 & 84.87 & Asymp. Sig. & 0 \\
\hline & Total & 195 & & & \\
\hline \multirow[t]{4}{*}{ Out in the Open* } & Undergraduate & 107 & 98.75 & Chi-Square & 18.542 \\
\hline & Graduate & 35 & 111.13 & df & 2 \\
\hline & Faculty & 51 & 83.64 & Asymp. Sig. & 0 \\
\hline & Total & 193 & & & \\
\hline \multicolumn{6}{|c|}{ Notes: *Significant at $P<.05$} \\
\hline
\end{tabular}

shops as worst places to study or work. Content analysis was done to cluster words with similar meaning that the participants had retrospectively described as why they considered those spaces as terrible. The top five words that qualified that made the space the worst place to work or study are noise, distractions, crowded, bad lighting, and lack of resources (see table 9). Items that that received fewer than five responses are not listed in the table.

Most occurrences of the word "library" referred to specific sections or floors of the Texas State University library or to libraries in which the noise level was not kept under control; however, some respondents did state that the library was their "least favorite place" because it is "too quiet." Additionally, respondents mentioned the library during "finals" and "construction" as a poor studying or working location due to excessive noise and crowds. The word "distractions" was often mentioned in relation to other individuals talking loudly or an excess of foot traffic in the study/work area, food court, and coffee shops. 


\begin{tabular}{|l|c|}
\hline \multicolumn{2}{|c|}{ TABLE 9 } \\
Attributes Used to Describe What Makes a Work or Study Space \\
TERRIBLE to Do Work (n = 165)
\end{tabular}

A total of 176 (88\%) individuals responded to the open-ended question about the best place they have studied or worked in and what made it so "great." The best places to study or do academic work included a variety of locations such as personal office spaces, home, coffee shop, as well as some floors and spaces of the library. Content analysis revealed that a space that is quiet, provided privacy, had resources, enabled one to focus or concentrate, had comfortable space and furniture, and had spacious work area/spread-out work space (see table 10) were the top six descriptors of what made a study/work space the best. Items that received fewer than five responses are not listed in the table.

\begin{tabular}{|l|c|}
\hline \multicolumn{2}{|c|}{ TABLE 10 } \\
Attributes Used to Describe What Makes a Work or Study Space GREAT to \\
Do Work (n = 176)
\end{tabular}


The word "room" also appeared in relation to the library, with respondents stating a preference for "private" study rooms because they are quiet and free of distractions; group rooms were also mentioned as positive locations free of distractions for collaborative work. Respondents also used the word "private" to describe areas where they could safely leave their belongings to move around when necessary without fear of items being stolen. "Library" and private office spaces were frequently mentioned as an ideal study/work location, although it was often accompanied by the qualifier that it must not be during finals week or while the building is under construction.

A total of $135(68 \%)$ individuals answered the open-ended question describing attributes that inspire them to study or work. Content analysis revealed that daylight, light/lighting, access to nature-outdoor views and plants, color, space and furniture that is comfortable, space that is quiet, some white noise, and access to resources were deemed as the top attributes they consider as inspiring in a space to study or work (see table 11). Items that received fewer than five responses are not listed in the table.

Respondents often used "comfortable" to describe both overall space as well as furniture. "Bright" referred to lights and colors in the space, and respondents often emphasized a need for bright enough light to read easily, but not too bright as to cause a glare. Respondents talked about the "colors" of the space in terms of their brightness/ warmth and the energy that they provide, as well as distaste for white walls given their "institutional" feel. Some respondents specifically mentioned blue-hued colors for calming purposes. Additionally, respondents expressed a need for plenty of "space" to spread out their work and provide them with the ability to move around. In terms of noise levels, many respondents expressed a need for "quiet," while some clarified

\begin{tabular}{|c|c|}
\hline \multicolumn{2}{|c|}{$\begin{array}{c}\text { TABLE } 11 \\
\text { Attributes Used to Describe Spaces that INSPIRE One to Study or Do } \\
\text { Academic Work }(n=135)\end{array}$} \\
\hline Attributes/Words & Frequency of Responses \\
\hline Daylight/Natural Light & 58 \\
\hline Light/Lighting & 50 \\
\hline Access to Nature (Outside Views/Plants/Water) & 47 \\
\hline Color & 34 \\
\hline Comfortable Furniture & 29 \\
\hline Quiet & 27 \\
\hline White Noise & 18 \\
\hline Resources & 18 \\
\hline Temperature & 15 \\
\hline Outside & 13 \\
\hline Food & 13 \\
\hline Plants & 12 \\
\hline Art & 10 \\
\hline Calm & 10 \\
\hline Work Space & 9 \\
\hline Clean & 8 \\
\hline Architectural Details & 8 \\
\hline
\end{tabular}


with statements such as "but not too quiet," indicating a need for some background noise based on personal preference.

\section{Discussion and Implications}

This exploratory survey indicates that faculty and students at Texas State University most often study/work alone compared to other study/work modes. To understand the library as a "third space," the current study focused on study and work spaces with no reference to library study spaces. Despite this, the desires for study/work spaces in the current study parallel those studies of both office spaces ${ }^{28}$ and library studies, such as Andrews University's student preference of individual study spaces over group and social areas. ${ }^{29}$ Respondents indicated that the types of work completed when working alone are studying, homework, research, and writing. These results, combined with the fact that Alkek Library has its largest door counts during the semester cycle when students most need quiet/silent spaces to do the types of work that they prefer to do alone, suggests that the library is seen as a place that provides those spaces. It may also indicate that quiet/silent individual study spaces cannot be found elsewhere on campus.

Study respondents also suggest a strong desire for quiet, private to semiprivate, individual space to work alone. Across work/study modes, "working out in the open" was considered least desirable, indicating that visual and acoustical privacy are important. To accommodate these needs, providing a combination of spaces, including space set aside for quiet/silent, private, individual work, is necessary within the larger learning commons design, something that is evident from this study's results as well as other recent studies. ${ }^{30}$ This suggests that literature predicting majority social-learning spaces in the learning commons may have swung too far in that direction, and this study and other most current literature reflect a correction.

Unwanted sound - noise - is an issue in many library studies. The study respondents also articulated this sentiment in their open-ended questions pertaining to study/work spaces. "Noise" appeared as the number one attribute that made a study/work space "terrible" to do work or study, while "quiet" was rated as the number one attribute that made a study/work space "great" to do work or study. The implementation of "quiet" and reduction of "noise" should be taken seriously in areas where traditional reading- and writing-based academic work/study occurs. There is ample evidence that noise is detrimental to cognitive task performance and concentration as well as increases stress and fatigue, ${ }^{31}$ affecting health and learning.

"Background noise" received low overall desirability ratings. However, the graduate students in general rated its desirability significantly higher than undergraduates and faculty in the work-alone and work-in-group mode. This has further implications for Alkek Library's renovations. In its quest to become a Carnegie Tier One Research University, the library must provide more support to graduate students. It is possible that certain areas in the library setting where working in groups or working alone with friends in a social setting tends to attract (and create) more noise. To counter this issue, the need for background noise or white noise to mask discernable sounds should be considered during renovation planning to provide acoustical privacy that will allow graduate students to work productively. Secure quiet space (with lockers) should be set aside to meet specific graduate student needs and encourage their use of the library as a study space of choice.

Respondents clearly voiced their opinions about what makes a study/work space inspiring and provided glimpses of both positive and negative attributes of work/ study spaces that affect a person's ability to study and work. Faculty and students want good indoor light/lighting, access to daylight and windows to provide a view. This is of significance as researchers are beginning to learn that the light (electric and 
daylight) not only affects vision and visual comfort, but the quantity (light level) and quality (spectrum and color) of light affects biological circadian aspects, attention, and cognitive aspects. ${ }^{32}$ Creating views to the outdoors has obvious implications for the design of new library/learning commons buildings. Creating these opportunities is more difficult in building renovations where the building envelope cannot be changed to provide more window/daylighting.

If daylight is not readily available, providing artificial or electric light that emulates daylight needs to be considered. With advances in LED and color-tunable LED technology, visual needs as well as biological needs can be easily met. ${ }^{33}$ Access to nature can also be achieved by use of small partitions with art, fish tanks, or screens with rhythmic movement, creating a "window" that gives the eye a place to rest during work breaks, and also helps to define a smaller, more enclosed space, increasing the sense of perceived privacy. ${ }^{34}$

The expressed desire for more quiet spaces introduced the question of crowding and noise, making the team take a cursory look at other spaces around campus. This led to an awareness of the constant background noise of everyday life on a crowded campus. Even if students did not have personal screens and devices, television screens dominate dorm common rooms and eating areas, moving digital signs attract attention, and the disrupting waves of chatter and movement of other students in crowded spaces generate visual and auditory distractions - all of which can create an undercurrent of stress for students. Because of this, the team sees the need for a study that includes a larger percentage of the undergraduate population, as this has implications for larger campus planning. It is possible that not all study spaces will exist within the library - at Texas State University or at other like institutions. On campuses where growth is not possible within the library structure itself, the team sees a trend toward providing smaller, "quiet study only" spaces across campus.

The team knows that attributes of space deeply affect both human performance and space use. In terms of library and learning commons space design, the team also foresees a trend in creating spaces where not just the space but the atmosphere-the lighting, noise levels, views, and sense of crowdedness - is planned to provide space for multiple learning styles and types of work, and particularly for quiet study. Gone will be the libraries that are like a single, large fast-food restaurant where the lighting is terrible, the furniture is fixed, and customers are required to be flexible. Instead, the team sees libraries adjusting the learning commons model to adapt large, open spaces into a series of boutique spaces, creating different atmospheres that support different kinds of work and learning styles. This will include appropriate amounts of silent and quiet individual study spaces, and it will be flexible enough to be modified by patrons to create the space they need, whether that is a group collaboration space or a small bubble of privacy and quiet in a public space.

Due to the time restrictions of the project, the team administered the survey during the summer, resulting in a small sample size and low response rate from undergraduate students. The implications of this are that the results cannot be generalized, nor can the team assume the survey captured all constituencies (first-generation and Hispanic students). This suggests a need for future studies that particularly consider user demographics, with a focus on undergraduate students. However, since this is an exploratory study, the results do lend direction to the planning committee and the architects designing study spaces in the library; the study also serves as a helpful addition to the team's other methods of gathering information (which are not part of this study).

The purpose of this study was to find what unique characteristics Texas State University students and faculty have regarding study modes, the type of work carried out when in each study mode, and what kinds of space attributes detract from - and 
inspire-study/work. While this study parallels others in types of space users' desire, it is unique in that the team did not limit the study to library spaces and that the survey specifically asked about spaces that provide inspiration. These descriptive results, examined against what the team knows about Texas State University's curricula and campus development, should help predict future needs within the library and larger campus. By understanding what attributes of space inspire students and faculty, and by removing the concept of library or learning commons, the team hopes to invite the architects to create designs that are responsive to user needs, even as the concept of the learning commons evolves. While these insights may be particular to Texas State University, these questions and this process may be applicable to other large, fastgrowing universities with limited library and campus space.

\section{Notes}

1. Arlee Turner, Bernadette Welch, and Sue Reynolds, "Learning Spaces in Academic Libraries: A Review of the Evolving Trends," Australian Academic E Research Libraries 44, no. 4 (2013): 226-34.

2. Jong-Ae Kim, “Dimensions of User Perception of Academic Library as Place," Journal of Academic Librarianship 42, no. 5 (2016): 509-14; Anne M. Houston, "Revisiting Library as Place," Reference \& User Services Quarterly 55, no. 2 (2015): 84-86; Susan E. Montgomery and Jonathan Miller, "The Third Place: The Library as Collaborative and Community Space in a Time of Fiscal Restraint," College \& Undergraduate Libraries 18, no. 2/3 (2011): 228-38.

3. "Best Colleges: Texas State University," U.S. News Higher Education website, available online at https://www.usnews.com/best-colleges/texas-state-university-3615/student-life [accessed 18 August 2018].

4. J.A. Franks and M.P. Tosko, "Reference Librarians Speak for Users: A Learning Commons Concept That Meets the Needs of a Diverse Student Body," Reference Librarian 47, no. 1 (2007): 105-18; Harlan Cleveland, "Editorial: How Can 'Intellectual Property' Be 'Protected'?" Change (1989): 10; JSTOR Journals, EBSCOhost [accessed 28 May 2017].

5. Scott Bennett, "Libraries and Learning: A History of Paradigm Change," portal: Libraries and the Academy 9, no. 2 (2009): 181-97; Kevin J. Fox, "The Evolution of the "Library as Place: An Analysis of Recent Academic Library Renovations" (UNC Chapel Hill thesis, January 2014): 1-36; Sharon Bostick and Bryan Irwin, "Library Design in the Age of Technology: Planning for a Changing Environment," IATUL Annual Conference Proceedings no. 35 (June 2014): 1-4.

6. Bostick and Irwin, "Library Design in the Age of Technology," 1-4.

7. Michael J. Khoo et al., "'A Really Nice Spot': Evaluating Place, Space, and Technology in Academic Libraries," College E Research Libraries 77, no. 1 (2016): 51-70.

8. Silas M. Oliveira, "Space Preference at James White Library: What Students Really Want," Journal of Academic Librarianship 42, no. 4 (2016): 355-67.

9. Fatt Cheong Choy and Su Nee Goh, "A Framework for Planning Academic Library Spaces," Library Management 37, no. 1/2 (2016): 13-28.

10. Choy and Goh, "A Framework for Planning Academic Library Spaces," 13-28; Daniel T. Willingham, Elizabeth M. Hughes, and David G. Dobolyi, "The Scientific Status of Learning Styles Theories," Teaching of Psychology 42, no. 3 (2015): 266-71; Susan Gordon-Hickey and Trey Lemley, "Background Noise Acceptance and Personality Factors Involved in Library Environment Choices by College Students," Journal of Academic Librarianship 38, no. 6 (2012): 365-69.

11. Oliveira, "Space Preference at James White Library," 355-67; Choy and Goh, "A Framework for Planning Academic Library Spaces," 13-28; Gordon-Hickey and Lemley, "Background Noise Acceptance," 365-69.

12. Gordon-Hickey and Lemley, "Background Noise Acceptance," 365-69.

13. "Learning Spaces - Libraries," Steelcase website, available online at https://www.steelcase. com/eu-en/discover/information/education-professionals/active-learning-spaces-libraries/ [accessed 18 August 2018].

14. Aram Seddigh et al., "Concentration Requirements Modify the Effect of Office Type on Indicators of Health and Performance," Journal of Environmental Psychology 38 (2014): 167-74; Jungsoo Kim and Richard de Dear, "Workspace Satisfaction: The Privacy-Communication TradeOff in Open-Plan Offices," Journal of Environmental Psychology 36 (2013): 18-26; Helena Jahncke et al., "Open-Plan Office Noise: Cognitive Performance and Restoration," Journal of Environmental Psychology 31, no. 4 (2011): 373-82; Jeremy Myerson, Jo-Anne Bichard, and Alma Erlich, New De- 
mographics, New Workspace: Office Design for the Changing Workforce (Aldershot: Gower Publishing, 2010).

15. Kim and de Dear, "Workspace Satisfaction," 18-26; A. Borisuit et al., "Effects of Realistic Office Daylighting and Electric Lighting Conditions on Visual Comfort, Alertness and Mood," Lighting Research \& Technology 47, no. 2 (2015): 192-209.

16. Oliveira, "Space Preference at James White Library," 355-67; Choy and Goh, "A Framework for Planning Academic Library Spaces," 13-28; Gordon-Hickey and Lemley, "Background Noise Acceptance," 365-69; Jahncke et al., "Open-Plan Office Noise," 373-82; Seung Hyun Cha and Tae Wan Kim, "What Matters for Students' Use of Physical Library Space?" Journal of Academic Librarianship 41, no. 3 (2015): 274-79; Janet E. Franks and Darla C. Asher, "Noise Management in Twenty-first Century Libraries: Case Studies of Four U.S. Academic Institutions," New Review of Academic Librarianship 20, no. 3 (2014): 320-31; Nancy J. Stone, "Designing Effective Study Environments," Journal of Environmental Psychology 21, no. 2 (2001): 179-90; Aram Seddigh et al., "The Effect of Noise Absorption Variation in Open-Plan Offices: A Field Study with a Cross-Over Design," Journal of Environmental Psychology 44 (2015): 34-44; Aram Seddigh et al., "The Association between Office Design and Performance on Demanding Cognitive Tasks," Journal of Environmental Psychology 42 (2015): 172-81; Valterri Hongisto et al., "Refurbishment of an Open-Plan Office: Environmental and Job Satisfaction," Journal of Environmental Psychology 45 (2016): 176-91.

17. Kim and de Dear, "Workspace Satisfaction," 18-26; Myerson, Bichard, and Erlich, New Demographics, New Workspace; Gregory A. Laurence, Yitzhak Fried, and Linda H. Slowik, "'My Space': A Moderated Mediation Model of the Effect of Architectural and Experienced Privacy and Workspace Personalization on Emotional Exhaustion at Work," Journal of Environmental Psychology 36 (2013): 144-52.

18. Laurence, Fried, and Slowik, "My Space," 144-52.

19. Albert Mehrabian and Shirley G. Diamond, "Effects of Furniture Arrangement, Props, and Personality on Social Interaction," Journal of Personality and Social Psychology 20, no. 1 (1971): 18-30.

20. Cagri Imamoglu and Meltem O. Gurel, “'Good Fences Make Good Neighbors': Territorial Dividers Increase User Satisfaction and Efficiency in Library Study Spaces," Journal of Academic Librarianship 42, no. 1 (2016): 65-73.

21. Oliver Keis et al., "Research Article: Influence of Blue-Enriched Classroom Lighting on Students' Cognitive Performance," Trends in Neuroscience and Education 3 (2014): 86-92.

22. P.J.C. Sleegers et al., "Lighting Affects Students' Concentration Positively: Findings from Three Dutch Studies," Lighting Research \& Technology 45, no. 2 (2013): 159-75; Claus Barkmann, Nino Wessolowski, and Michael Schulte-Markwort, "Applicability and Efficacy of Variable Light in Schools," Physiology \& Behavior 105 (2012): 621-27.

23. Lisa Heschong, Roger L. Wright, and Stasia Okura, "Daylighting Impacts on Human Performance in School," Journal of the Illuminating Engineering Society 31, no. 2 (2002): 101-14.

24. Borisuit et al., "Effects of Realistic Office Daylighting," 192-209.

25. Didem Kan Kilic and Deniz Hasirci, "Daylighting Concepts for University Libraries and Their Influences on Users' Satisfaction," Journal of Academic Librarianship 37, no. 6 (2011): 471-79.

26. "Learning Spaces-Libraries," Steelcase website.

27. MIT Libraries Survey (2014), available online at http://libguides.mit.edu/ld.php?content_ id $=13753638$ [accessed 18 August 2018].

28. Seddigh et al., "Concentration Requirements," 167-74; Kim and de Dear, "Workspace Satisfaction," 18-26; Jahncke et al., "Open-Plan Office Noise," 373-82; Myerson, Bichard, and Erlich, New Demographics, New Workspace; Borisuit et al., "Effects of Realistic Office Daylighting," 192-209; Seddigh et al., "The Effect of Noise Absorption," 34-44; Seddigh et al., "The Association between Office Design and Performance," 172-81; Laurence, Fried, and Slowik, "My Space," 144-52.

29. Khoo et al., "A Really Nice Spot," 51-70; Oliveira, "Space Preference at James White Library," 355-67; Cha and Kim, "What Matters for Students' Use of Physical Library Space?" 274-79.

30. Khoo et al., "A Really Nice Spot," 51-70; Cha and Kim, "What Matters for Students' Use of Physical Library Space?" 274-79; Francine May and Alice Swabey, "Using and Experiencing the Academic Library: A Multisite Observational Study of Space and Place," College \& Research Libraries 76, no. 6 (2015): 771-95.

31. Willingham, Hughes, and Dobolyi, "The Scientific Status of Learning Styles Theories," 266-71; Seddigh et al., "Concentration Requirements," 167-74; Jahncke et al., "Open-Plan Office Noise," 373-82; Seddigh et al., "The Effect of Noise Absorption," 34-44; Seddigh et al., "The Association between Office Design and Performance," 172-81; Hongisto et al., "Refurbishment of an Open-Plan Office," "176-91.

32. Borisuit et al., "Effects of Realistic Office Daylighting," 192-209; M.B.C. Aries, M.P.J. Aarts, and J. van Hoof, "Daylight and Health: A Review of the Evidence and Consequences for the Built Environment," Lighting Research \& Technology 47, no. 1 (2015): 6-27; L. Bellia, A. Pedace, and G. 
Barbato, "Lighting in Educational Environments: An Example of a Complete Analysis of the Effects of Daylight and Electric Light on Occupants," Building \& Environment 68 (2013): 50-65.

33. Barkmann, Wessolowski, and Schulte-Markwort, "Applicability and Efficacy of Variable Light in Schools," 621-27; Michael S. Mott et al., "Illuminating the Effects of Dynamic Lighting on Student Learning," SAGE Open 2, no. 2 (2012): 1-9.

34. Seddigh et al., "Concentration Requirements," 167-74; Kim and de Dear, "Workspace Satisfaction," 18-26; Laurence, Fried, and Slowik, "My Space," 144-52. 